\title{
Diseño de polinizador mediante vibración forzada en invernaderos tipo túnel
}

\section{Pollinator design through forced vibration in tunnel type green houses}

\author{
LÓPEZ-MARTÍNEZ, Víctor Manuel†* \\ Universidad Politécnica de Atlacomulco \\ ID $1^{\text {er }}$ Autor: Victor Manuel, López-Martínez / ORCID: 0000-0001-5172-3514, CVU CONACYT ID: 456578
}

DOI: $10.35429 /$ JTEN.2019.12.3.1.7

Recibido 04 de Octubre, 2019, Aceptado, 03 de Diciembre, 2019

\begin{abstract}
Resumen
Ante la problemática de la decreciente población de las abejas a nivel global, se requiere implementar medidas que contribuyan tanto a la protección de estos insectos polinizadores y el desarrollo de sistemas de polinización asistida (Roubik, 1989). Una alternativa a los cultivos de ciertas especies es mediante el uso de invernaderos que permiten un mejor ambiente para la especie en cuestión. Pero en la actualidad los invernaderos para plantas de enredadera en el cual se centra el presente trabajo de investigación, no tienen una polinización que permita una alta eficiencia en su proceso. Para lo cual se propone el uso de métodos artificiales para lograr una polinización asistida con base en el uso de la vibración forzada y por convección que permita optimizar la producción. El diseño consiste en elementos que produzcan oscilaciones vibratorias, de manera que el polen sea dispersado entre las plantas, logrando así una producción más óptima. Se presentan los fundamentos de alcance de frecuencias a desarrollar por el dispositivo de vibración y el modelo del sistema. Así como los parámetros y variables físicas que intervienen en el proceso, pretendiendo así aportar una alternativa que permita lograr una polinización efectiva ante la inminente baja de la población de las especies polinizadoras.
\end{abstract}

Polinización, Invernadero, Vibración

\begin{abstract}
Given the problem of the declining population of bees globally, it is necessary to implement measures that contribute both to the protection of these pollinating insects and the development of assisted pollination systems (Roubik, 1989). An alternative to the cultivation of certain species is through the use of greenhouses that allow a better environment for the species in question. But at present the greenhouses for creeper plants in which the present research work is focused, do not have a pollination that allows a high efficiency in their process. For which the use of artificial methods is proposed to achieve assisted pollination based on the use of forced vibration and convection that allows production to be optimized. The design consists of elements that produce vibratory oscillations, so that the pollen is dispersed among the plants, thus achieving a more optimal production. The fundamentals of frequency range to be developed by the vibration device and the system model are presented. As well as the parameters and physical variables involved in the process, thus seeking to provide an alternative that allows for effective pollination in the face of the imminent decline in the population of pollinator species.
\end{abstract}

Pollination, Greenhouse, Vibration

Citación: LÓPEZ-MARTÍNEZ, Víctor Manuel. Diseño de polinizador mediante vibración forzada en invernaderos tipo túnel. Revista de Ingeniería Tecnológica. 2019 3-12: 1-7

\footnotetext{
* Correspondencia del Autor (Correo electrónico: victor_lopez@cccti.com.mx)

$\dagger$ Investigador contribuyendo como primer autor.
} 


\section{Introducción}

Una forma de polinización es el proceso en el cual los insectos revolotean de flor en flor llevando involuntariamente polen desde los alambres de una flor hasta alcanzar el estigma de esa misma $u$ otra flor, en principio de la misma especie (Thomas 2012). Este transporte de polen se lleva a cabo desde los estambres (órganos florales masculinos) de una flor hacia los estigmas (órganos florales femeninos) de otra flor, dónde luego se produce la germinación y fecundación, haciendo posible para producción de semillas y frutos (Sygenta) como se ilustra en la figura 1 .

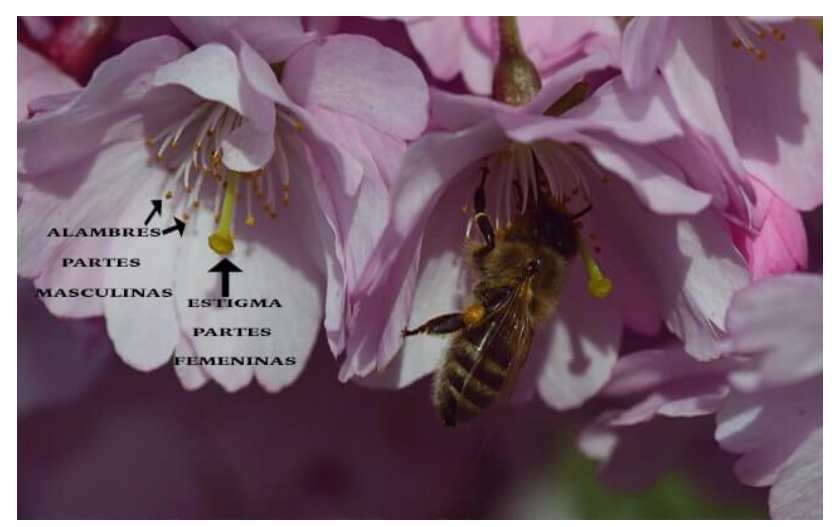

Figura 1 Partes de la flor para ser polinizada

Fuente: https://generacionverde.com/blog/ambiental/quees-la-polinizacion

\section{Los agentes polinizantes naturales: Insectos, viento y agua}

\section{Insectos polinizadores}

Los animales, sobre todo los insectos en nuestras latitudes juegan un papel vital en la reproducción de las plantas al facilitar la polinización de muchas especies vegetales. Estos polinizadores, en especial los insectos abarcan un gran número de especies ya que estos animales tienen una manera muy sencilla y rápida de reproducirse a lo contrario de un murciélago, pero aun así también existen algunos reptiles como, aves, e incluso mamíferos (como los lémures) que también pueden desempeñar esta importante labor sin importar las ventajas o desventajas que tienen (Generación verde 2016).

$$
\text { A continuación se presentan } 7
$$
polinizadores más comunes:

$$
\begin{array}{ll}
- & \text { Las abejas } \\
- & \text { Las avispas } \\
- & \text { Las hormigas }
\end{array}
$$

$$
\begin{array}{ll}
- & \text { Las moscas } \\
- & \text { Las mariposas } \\
- & \text { Los colibríes } \\
- & \text { Los murciélagos }
\end{array}
$$

\section{Polinización por viento}

Muchas especies vegetales están adaptadas a la polinización por el viento (plantas anemófilas), por ejemplo las ortigas, alisos, robles, abedules, hayas, la mayoría de las coníferas o las gramíneas. Los granos de polen de estas plantas se producen en grandes cantidades y suelen ser pequeños y/o secos, fáciles de transportar por el viento.

\section{Polinización por agua}

Aunque menos frecuente, está bien desarrollada en plantas de agua dulce, como las lentejas de agua, así como en las especies del género Zostera (de aguas salobres), cuyos granos de polen son liberados en masa y transportados (Sygenta).

Los granos de polen de las coníferas poseen un par de sacos de aire, que se asocian con la aerodinámica y la hidrodinámica, porque le permiten al polen ser transportado con éxito por el aire o por el agua (Generación verde 2016).

\section{Polinización por vibración (zumbido)}

La polinización por zumbido o polinización vibratoria es una técnica usada por ciertas abejas y abejorros para extraer polen de aquellas flores que requieren de este proceso (Fidalgo 2009). Las anteras de las plantas que son polinizadas por medio de vibraciones suelen ser tubulares con una abertura en el extremo. A diferencia de la dehiscencia de la mayoría de las anteras, el polen permanece dentro de ella y sale solo por el poro. En el caso de algunas plantas autogámicas (que se autopolinizan) como el tomate el viento puede bastar para mover los granos de polen y realizar polinización. Pero la actividad vibratoria de las abejas asegura un grado más elevado de polinización. Para aflojar el polen, la abeja se aferra a la flor y mueve rápidamente sus músculos alares, sin mover las alas. Esto produce un zumbido con un sonido característico; esta vibración afloja los granos de polen y hace que emerjan de la antera en la figura 2 se ilustra este proceso. 
La abeja doméstica no es capaz de efectuar esta operación y no puede polinizar ciertas plantas (Thomas 2012).

Más de 70 familias y alrededor de 20,000 especies de plantas, es decir aproximadamente el 9\% de las flores, son polinizadas primariamente por medio de polinización vibratoria (Buchmann 1985).

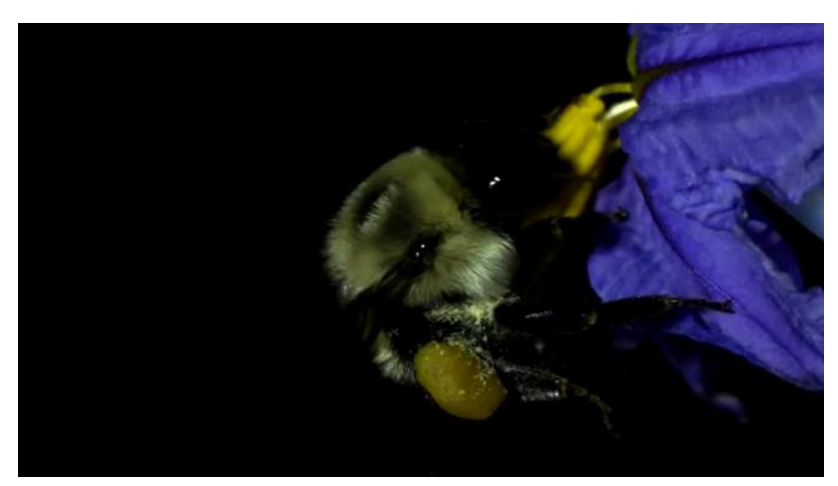

Figura 2 Polinización vibratoria de abeja

Fuente: Karl Foord, Universidad de Minessota.

En la figura 3 se muestra el espectro de frecuencias de una vibración floral generada por una abeja Bombus audax, el pico más alto re resenta la más alta amplitud relativa de $1 \mu \mathrm{m}$ a una frecuencia dominante que oscila entre los $300-400 \mathrm{~Hz}$ que vendría siendo la frecuencia fundamental de vibración con la cual el polen es liberado desde las anteras que es donde se produce el polen (Arroyo-Correa 2018).

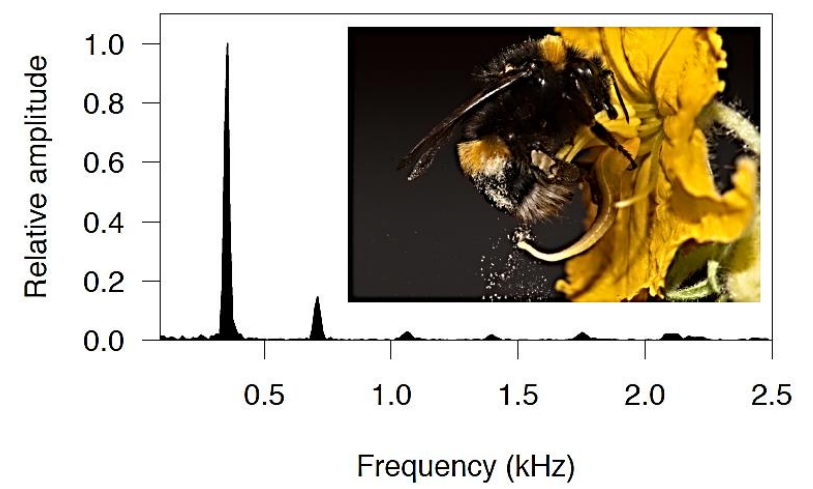

Figura 3 Frecuencia y amplitud $(\mu \mathrm{m})$ de vibración generada por la abeja polinizadora

Fuente: Arroyo-Correa (2018)

\section{El problema de producir en invernadero}

Los animales polinizadores viven al aire libre, no en invernaderos. Entonces, o se poliniza a mano o mediante un método de polinización artificial como los tratados previamente o se introduce el insecto polinizador en este caso la abeja, y eso genera problemas, como ya ocurre con el jitomate.
Esta hortaliza en invernadero rinde y produce ganancias, pero su polinizador, el tipo de abeja, generalmente proviene la Unión Europea y se vende en contenedores. Se introduce al invernadero, pero es muy fácil que se escape al abrir la puerta o por ranuras o agujeros del plástico; entonces empieza a desplazar a las especies nativas. México lo importa de Holanda, porque es más fácil comprarlo que atraparlo, lo cual desplaza a las especies nativas y estos agentes polinizadores además de que requieren un medio abierto, pueden traer consigo enfermedades y bacterias que pueden ser transmitidas a los cultivos dentro del invernadero (Del Coro 2009).

\section{Polinización artificial}

La polinización artificial consiste en la utilización de métodos donde se requiere la intervención del hombre, estos van desde el uso de elementos mecánicos como ventiladores, sistemas de riego, con los cuales se logra desprender el polen de la flor, hasta métodos más rudimentarios como el sacudimiento de las plantas con varas o manualmente. Tambien existen los llamados "electric bees" que son motores eléctricos a altas frecuencias que hacen vibrar la flor y así poder extraer el polen de la misma (Generación verde 2016). Si bien estos métodos han sido provechosos hasta cierto punto, no son los más eficientes que se puedan emplear, dado que es común afectar a la planta en su estructura anatómica, dado que inevitablemente se realizan movimientos bruscos a la misma ya sea por contacto directo o por medio de corrientes ya sean de agua o de viento a altas velocidades y aun así no se garantiza una polinización adecuada.

Dadas estas deficiencias de polinización artificial aunadas al problema que se avecina de la disminución de las poblaciones de los agentes polinizadores naturales, surge la necesidad de desarrollar nuevas técnicas de polinización que sean lo menos invasivas hacia la planta y que permitan una polinización eficiente. Para lo cual se propone un método artificial que abarca las formas de polinización antes descritas pero cuidando al máximo la afectación a la planta.

La desaparición de los polinizadores significa un riesgo para la producción de alimentos. 
Las abejas, mariposas, colibrís y murciélagos, principales animales de ese tipo en el país, son necesarios para $90 \%$ de las plantas del planeta. La agricultura intensiva, la insistencia en el uso de plaguicidas y las especies exóticas invasoras han comenzado a poner en riesgo la vida de los polinizadores, animales encargados de unir el gameto masculino con el femenino y facilitar la fertilización de las plantas (Del Coro 2009). Aunque en México, debido a su extraordinaria biodiversidad, aún no se han presentado señales claras de que las poblaciones de esos ejemplares estén desapareciendo, en países como Estados Unidos ya existen graves problemas. Ante esa situación se considera que nuestro país debe emprender acciones al respecto, porque la extinción de los polinizadores no sólo significaría el fin de unos servidores ambientales indispensables, sino pondría en riesgo la producción de alimentos, y con ella, la supervivencia de la humanidad. Dentro de los principales polinizadores en México se encuentran las abejas, luego las mariposas, los colibríes y los murciélagos (Del Coro 2009).

\section{Metodología}

El método consiste en asimilar las frecuencias vibratorias que genera una abeja al posarse sobre la flor, tales frecuencias tienen que oscilar alrededor de los $400 \mathrm{~Hz}$ y generar una amplitud de onda de alrededor de $1 \mu \mathrm{m}$. Para el tema del transporte del polen, como se lleva a cabo en la mayoría de las plantas de manera natural, es mediante corrientes de aire que sirven como medio de transporte generando corrientes convectivas del mismo generando velocidades de masa de aire a un máximo de $30 \mathrm{~km} / \mathrm{hr}$ que llevándolas de manera intermitente dentro de un invernadero, estas no afectan ni la estructura de la planta ni a la misma flor. Dichas pruebas se llevaron a cabo mediante la simulación previa en un software CAD.

En la figura 4 se proponen dimensiones próximas a un invernadero real y se muestran las corrientes de aire bajo la configuración propuesta de las entradas de aire laterales a una velocidad mínima de $0.5 \mathrm{~m} / \mathrm{s}$ hasta una máxima de $8 \mathrm{~m} / \mathrm{s}$ y los orificios de salida superior del invernadero, generando así corrientes convectivas que propician el transporte del polen procurando sean en todas las direcciones del mismo.

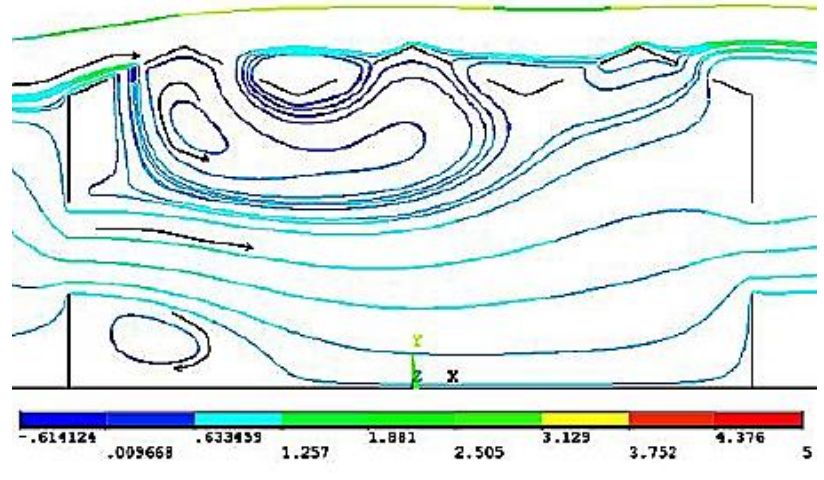

Figura 4 Invernadero con corrientes convectivas de aire corte frontal

Respecto al sistema de vibración se propone un oscilador de alta frecuencia como las alcanzadas por la mayoría de las abejas que es de aproximadamente $400 \mathrm{~Hz}$, se pretende colocar el sistema de oscilación en partes estratégicas de los alambres tensores de los invernaderos como se ilustra en la figura 5, dado que la propuesta está centrada para ser operada en sistemas cerrados. Los sistemas de oscilación permanecerán instalados de manera estática con sistemas de transmisión de movimientos oscilatorios a través de las cuerdas de sujeción de las enredaderas de la plantas, llevando así las oscilaciones a la frecuencia necesaria requerida para el desprendimiento del polen y en conjunto con el sistema convectivo de corrientes de aire, transportar el polen hacia todos los espacios dentro del invernadero para que se pueda llevar a cabo la polinización de una manera más eficiente.

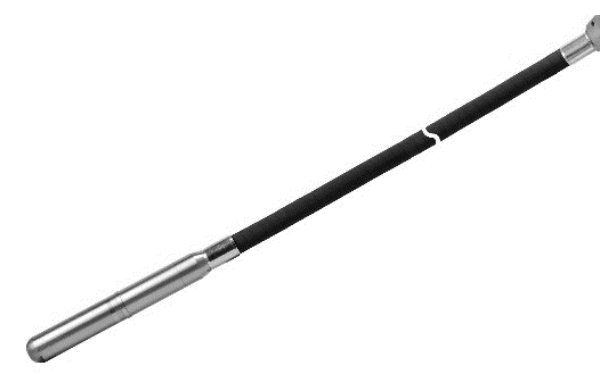

Figura 5 Configuración propuesta de los sistemas de oscilación

El modelado del sistema corresponde a un sistema de vibración forzada amortiguada donde la fuerza de excitación y el coeficiente amortiguación son constantes, se muestra la ecuación general de un sistema con estas características:

$m \ddot{z}+\gamma \dot{z}+k z=-m g$ 
La solución para el caso estacionario es:

$$
m \cdot 0+\gamma \cdot 0+k z_{s}=-m g \quad \Rightarrow \quad z_{s}=-\frac{m g}{k}
$$

En este caso se tiene una fuerza excitadora sinusoidal entonces se tiene:

$$
F(t)=F_{0} \cos (\omega t)
$$

Mediante el uso de fasores se tiene el valor de la amplitud el sistema:

$$
A=|\hat{x}|=\frac{F_{0} / m}{\sqrt{\left.\left(\omega_{0}^{2}-\omega^{2}\right)^{2}+4 \beta^{2} \omega^{2}\right)}}
$$

Para el sistema propuesto se sustituyen los valores y de esta manera se procede a validar si la amplitud resultante es la requerida:

$$
\begin{aligned}
& \text { Fo }=200 \mathrm{~N} \\
& \omega_{\mathrm{o}}=4.48 \mathrm{rad} / \mathrm{s} \\
& \omega=2513 \mathrm{rad} / \mathrm{s}
\end{aligned}
$$

Sustituyendo los valores en la ecuación la amplitud A se obtiene:

$$
\mathrm{A}=1.5 \times 10^{-6} \mathrm{~m}
$$

$\mathrm{Si}$ consideramos que se requiere una amplitud de $1 \mu \mathrm{m}$ para alcanzar a liberar el polen de la antera de la flor, se tiene que con los valores iniciales propuestos en la ecuación de amplitud, son suficientes para lograr llevar a la planta la frecuencia de vibración requerida para lograr tal efecto.

\section{Resultados}

Se desarrolló el modelo del prototipo en CAD para ilustrar el sistema previo a una posible implementación, se representa el alambrado del invernadero y como es que influye en el desarrollo de la planta, ya que es de donde ésta se sostiene para poder continuar con su crecimiento. Inicialmente se tiene un arreglo de alambres transversal a lo largo del invernadero y posteriormente se desprende una red secundaria que es donde descienden los alambres de la enredadera como se ilustra en la figura 6 .

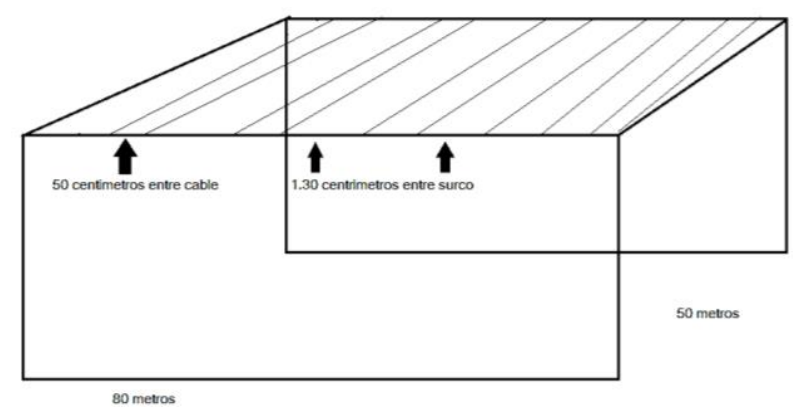

Figura 6 Configuración típica de un invernadero de jitomate

En la figura 7 se muestra el esquema del sistema polinizador propuesto el cual consta de bandas rotatorias mediante un motor que genera la frecuencia necesaria para el desprendimiento del polen en la planta.

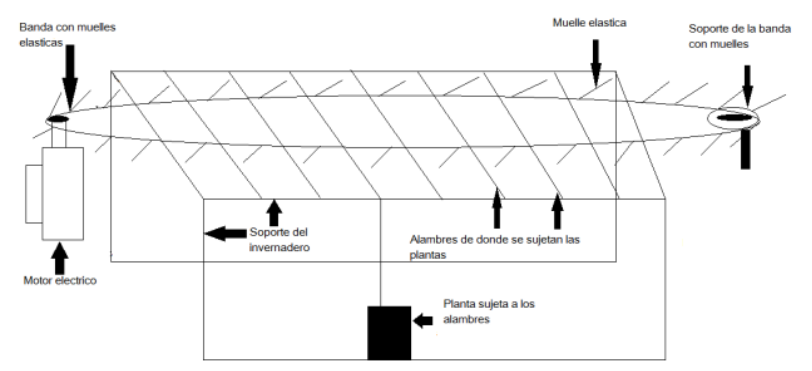

Figura 7 Sistema vibratorio propuesto

El diseño se basa en la adición de "uñas" a la malla de alambre para que rasguen estos como si fuera una guitarra, $y$ de esta forma generar oscilaciones que se transfieran en toda la red hasta llegar a la planta, como se muestra en el corte longitudinal en la figura 8.

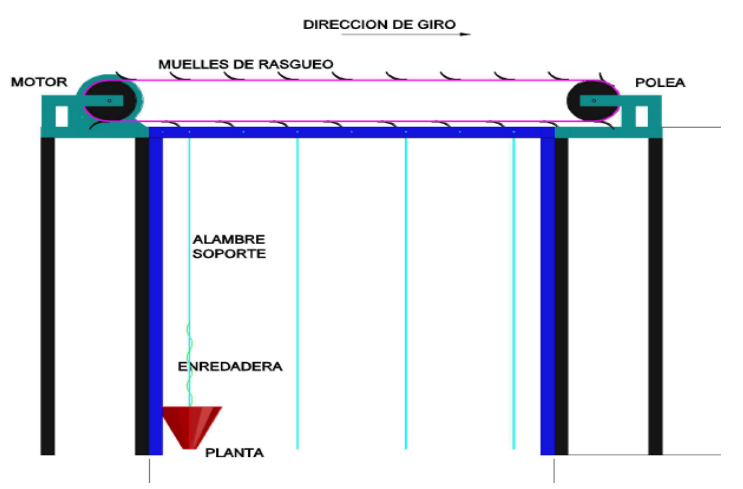

Figura 8 Corte longitudinal del sistema

El modelo virtual propuesto en la figura 9 y 10 es lo que se pretende implementar en un invernadero de jitomate real para realizar pruebas en situ del sistema propuesto y validar la efectividad del mismo, por lo que el alcance hasta este punto es únicamente de diseño y fundamentación del sistema. 


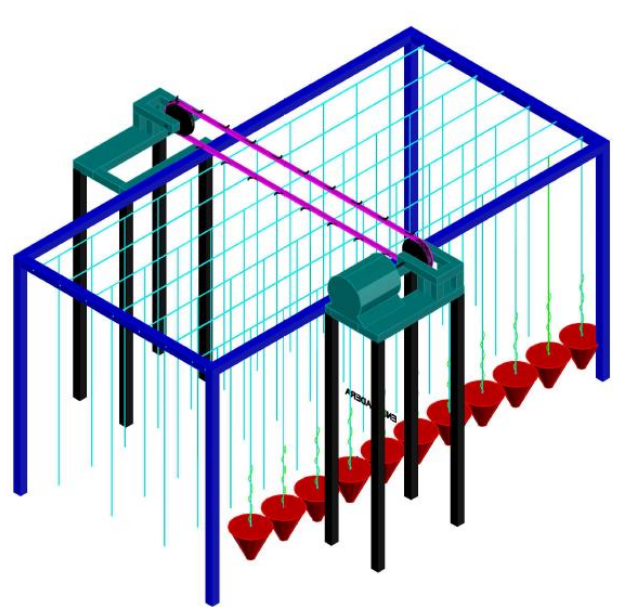

Figura 9 Vista en perspectiva del modelo propuesto. Fuente: el autor

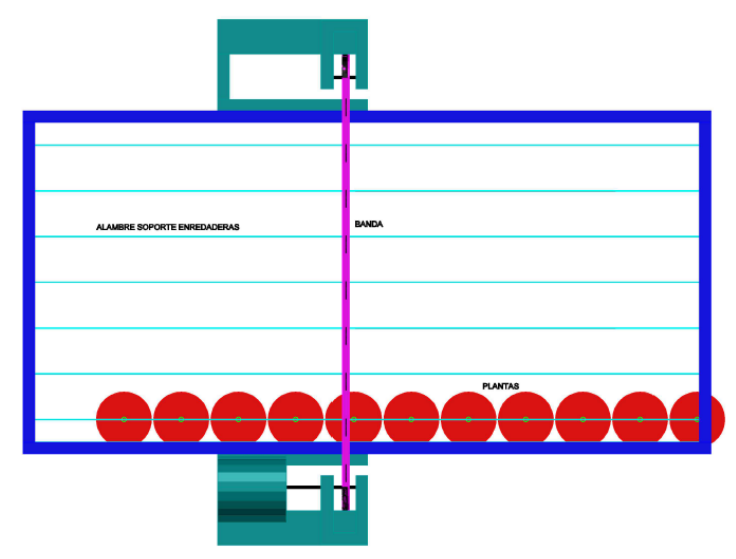

Figura 10 Vista en perspectiva del modelo propuesto

\section{Conclusiones}

El trabajo con sistemas sustentables ha sido un tema de gran importancia dada la situación actual en lo que a la disminución de agentes polinizadores se refiere, diversos estudios sugieren que el uso de diversas radiofrecuencias provocan desorientación de las abejas y otras especies, contribuyendo al desbalance del tema de la polinización, en este trabajo se trató un tema de carácter de ingeniería mecánica y de control con lo cual se pretendió dar un aporte como alternativa a los sistemas de polinización el cual retoma en mayor o menor medida como se puede implementar cada uno de ellos mediante convección y vibración forzada.

Las propuestas de diseño desde el inicio del presente tema de investigación han ido cambiando y no se descartan posibles modificaciones futuras, lo que da pauta a que el tema da para mejoras subsecuentes.
Las generación de las corrientes convectivas de aire es un parámetro crucial a considerar sobre todo al considerar las entradas y salidas de aire al invernadero ya que el objetivo fue lograr la máxima dispersión de aire con polen para lograr una polinización cruzada efectiva, la simulación virtual permitió modelar tales entradas y salidas de aire con un flujo aceptable aunque con corrientes de aire de bajas velocidades lo cual no fue muy apropiado debido a que bajas velocidades no garantizan un transporte de polen efectivo debido al peso del mismo.

El modelado de las vibraciones mecánicas es un tema que requiere más análisis dado que no se toman en cuenta las armónicas y los coeficientes de amortiguación de los materiales empleados por lo que el profundizar en el análisis de las pérdidas por histéresis podría arrojar más datos para la mejora del sistema y así llegar a los parámetros esperados mediante los fundamentos teóricos.

Aun así el sistema propuesto es un inicio para futuras mejoras $\mathrm{y}$ adecuaciones que permitan lograr su cometido de lograr una polinización más eficiente, ya que como se explicó previamente, los métodos utilizados actualmente para polinizar de manera artificial son rudimentarios pero con la certeza de que a pesar de sus limitantes funcionan.

El proyecto se sigue trabajando y mejorando, se pretende realizar pruebas físicas en una siguiente etapa ya que existe acercamiento con productores de jitomate de la zona interesados en la propuesta por lo que el proyecto mantiene una continuidad tanto a nivel investigación y próximamente en campo con adquisición de información in situ.

\section{Referencias}

Syngenta. Polinizadores. Recuperado el 12 de agosto de 2019 de: https://polinizadores.com/polinizacion/que-esla-polinizacion/

Generación verde (2016). ¿Qué es la polinización? Recuperado el 10 de agosto del 2019 de: https://generacionverde.com/blog/ambiental/qu e-es-la-polinizacion 
Fidalgo, Adriana De O.; Kleinert, Astrid De M. P. (2009). Reproductive biology of six Brazilian Myrtaceae:-is there a syndrome associated with buzz-pollination?. Recuperado el 11 de agosto del 2019 de: https://dx.doi.org/10.1080/0028825x.2009.9672 71

Thomas S. Woodcock (2012), Pollination in the Agricultural Landscape: Best Management Practices for Crop Pollination. Recuperado el 10 de Agosto del 2019 de: http://www.pollinator.ca/bestpractices/images/P ollination\%20in\%20Agricultural\%20Landscape _Woodcock

_Final.pdf, Canadian Pollination Initiative (NSERC-CANPOLIN)

Buchmann, Stephen L. (1985). Bees Use Vibration to Aid Pollen Collection from NonPoricidal Flowers. Journal of the Kansas Entomological Society 58 (3): 517-525. JSTOR 25084671. Recuperado el 7 de agosto del 2019 de: https://www.jstor.org/stable/250846

71.

Blanca Arroyo-Correa, Ceit Beattie1, Mario Vallejo-Marín (2018). Bee and floral traits affect the characteristics of the vibrations experienced by flowers during buzz-pollination. Journal of Experimental Biology doi: 10.1242/jeb.198176. Recuperado el 30 de julio del 2019 de: https://www.biorxiv.org/content/10.1101/49469 0v1

Del Coro (2009). La desaparición de los polinizadores, riesgo para la producción de alimentos. Periódico la Jornada. Recuperado el 22 de Julio del 2019 de: https://www.jornada.com.mx/2009/01/13/index. php? section $=$ ciencias $\&$ article $=\mathrm{a} 02 \mathrm{n} 1$ cie 